\section{Relação entre violência física, consumo de álcool e outras drogas e bullying entre adolescentes escolares brasileiros}

\author{
Association between physical violence, consumption \\ of alcohol and other drugs, and bullying among \\ Brazilian adolescents
}

1 Faculdade de Saúde Pública, Universidade de São Paulo, São Paulo, Brasil. 2 Secretaria de Vigilância em Saúde, Ministério da Saúde, Brasília, Brasil.

Correspondência S. S. C. A. Andrade Faculdade de Saúde Pública, Universidade de São Paulo. Rua Oscar Freire 2040, apto. 111, São Paulo, SP 05409-011, Brasil silvania.andrade@usp.br

\begin{abstract}
This study aimed to identify the association between alcohol and drug consumption and bullying on the one hand and involvement in situations of physical violence among adolescents 13 to 15 years in public and private schools in State capitals and the Federal District of Brazil. The study analyzed data from the National School Health Survey (PeNSE) for the year 2009. Data analysis used logistic regression. Prevalence of involvement in physical violence was $12.9 \%$ more common in boys than girls. Both genders showed associations between physical violence or being a victim of bullying and use of illegal drugs, plus the heightened effect of the combined consumption of alcohol and other drugs. In boys, alcohol consumption showed a significant association with physical violence. Having the father or both parents living at home was inversely associated with physical violence in girls. Knowledge of factors associated with physical violence among adolescents is important for supporting health promotion strategies and a culture of peace, thereby counteracting the idea of taking teenage violence for granted.
\end{abstract}

Violence; Adolescent; Alcohol Drinking; Street Drugs; Bullying
Silvania Suely Caribé de Araújo Andrade 1 Renata Tiene de Carvalho Yokota ${ }^{2}$ Naíza Nayla Bandeira de Sá 2 Marta Maria Alves da Silva 2 Wildo Navegantes de Araújo 2 Márcio Dênis Medeiros Mascarenhas 2 Deborah Carvalho Malta 2

A violência é definida como uso intencional da força física ou do poder, de modo real ou em ameaça, autoinflingida, interpessoal ou coletiva, que resulte ou tenha alta probabilidade de resultar lesão, óbito, dano psicológico, deficiência de desenvolvimento ou privação. $\mathrm{O}$ ato violento pode ser de natureza física, sexual, psicológica ou privação/negligência. A violência interpessoal subdivide-se em violência familiar e violência comunitária. O local de ocorrência mais frequente de situações de violência familiar é o domicílio, entretanto a violência comunitária pode ocorrer na via pública ou em instituições como a escolar 1 .

A violência física é um tipo de violência comum entre adolescentes independentemente da cultura 2 . Em relação à violência no ambiente escolar, uma pesquisa realizada em 41 países da América do Norte e Europa, nos anos de 2005 e 2006, constatou que $14 \%$ dos adolescentes estudados relataram envolvimento em pelo menos três brigas nos 12 meses que antecederam a entrevista, proporção maior entre meninos do que em meninas ${ }^{3}$. No mundo, a prevalência de violência física entre adolescentes escolares é diversa, variando de $31,2 \%$ em Portugal a 45,5\% em Israel, no período de um ano 4,5 .

No Brasil, as causas externas apresentam-se como agravos de maior impacto na morbimortalidade de adolescentes, com tendência crescente na mortalidade relacionada à violência a partir dos 15 anos 6,7,8,9. A prevalência de atendimentos 
relativos à violência foi de $18,7 \%$ na faixa etária de 10 a 19 anos, segundo dados do Inquérito de Vigilância das Violências e Acidentes (VIVA 2009), realizado em serviços sentinelas de urgência e emergência de 23 capitais e no Distrito Federal 10. Existe uma associação entre comportamentos agressivos e vitimização na adolescência e violência doméstica 11,12,13, sendo a violência física o tipo mais frequente de violência doméstica contra crianças e adolescentes 14 . Um estudo feito em um município da Região Metropolitana de São Paulo, revelou uma prevalência de punição física grave de crianças/adolescentes por um ou ambos os pais de $20 \%$ no período de 12 meses 13. Foi observada prevalência de violência física severa perpetrada pelos pais em pelo menos uma vez na vida da magnitude de $14,6 \%$ entre adolescentes escolares de São Gonçalo (Rio de Janeiro) 12.

Aproximadamente $71 \%$ dos adolescentes de escolas públicas selecionadas em Porto Alegre (Rio Grande do Sul) afirmaram terem sido vítimas de violência física na comunidade; a situação mais frequente relatada foi assaltos 15 . Um inquérito realizado com adolescentes escolares da Região Metropolitana de São Paulo verificou que a prevalência de envolvimento em brigas com agressão física mais de duas vezes nos últimos 12 meses anteriores à pesquisa foi de 15,2\% entre os adolescentes estudantes da rede pública de ensino, e de $20,3 \%$ entre aqueles da rede privada 16. Estudos locais estimam que a prevalência de violência física entre adolescentes brasileiros foi de aproximadamente $22,8 \%$ para participação em brigas com agressão, sendo maior no sexo masculino 2 .

Consumo de bebidas alcoólicas, uso de drogas ilícitas, tabagismo, sedentarismo, violência familiar, falta de supervisão dos pais, bullying, evasão e reprovação escolar, autoestima diminuída e baixo nível socioeconômico são fatores de risco relacionados a situações de violência física entre os adolescentes 2,3,17,18,19,20,21,22. O consumo de álcool e drogas ilícitas entre os adolescentes apresenta associações similares àquelas citadas para o envolvimento em situações de violência física 18,23,24,25. A distribuição desses fatores é distinta entre meninos e meninas, sendo que os comportamentos de risco são mais frequentes no sexo masculino 2,3,5,18,23,25,26. Religiosidade, supervisão familiar, relacionamento satisfatório com os pais, bom desempenho escolar e integração na escola foram identificados como fatores de proteção para os adolescentes em relação à exposição a situações de violência física entre os pares $3,18,19,20,24,25,27,28$.

A adolescência é uma fase de aquisição de hábitos saudáveis, bem como de exposição a situações de risco com efeitos significativos no presente e no futuro ${ }^{6}$. Nesse contexto, a escola também é considerada como um ambiente de integração social e de estímulo ao desenvolvimento e pode ser um espaço de exposição a situações de violência. Ressalta-se que a violência apresenta um impacto na saúde mental dos adolescentes, sendo considerada o fator de risco mais importante para problemas relativos à saúde mental de escolares 29. A Organização Mundial da Saúde (OMS) tem priorizado o monitoramento nessa faixa etária e tem realizado inquéritos como o Global School-based Student Health Survey para estudo dos comportamentos e fatores de risco e proteção entre adolescentes 30 .

Em vista da importância do monitoramento da população escolar e do número insuficiente de pesquisas nacionais sobre o tema e o caráter local dos estudos realizados 2,17, o Instituto Brasileiro de Geografia e Estatística (IBGE) e o Ministério da Saúde realizaram, em 2009, a Pesquisa Nacional de Saúde do Escolar (PeNSE) 6, visando a contribuir no diagnóstico de fatores de risco e de proteção para a saúde dos adolescentes. Neste trabalho também foi incluído um módulo para estudar as situações de violência vividas por estudantes adolescentes. O objetivo do presente estudo foi identificar a associação entre o consumo de álcool e outras drogas e o bullying com o envolvimento em situações de violência física entre adolescentes em escolas públicas e privadas.

\section{Métodos}

\section{Tipo de estudo}

Estudo de análise de dados provenientes da PeNSE 6 do ano de 2009. A PeNSE é um inquérito epidemiológico trienal (o primeiro foi feito em 2009) realizado com adolescentes de 13-15 anos do 9‥ ano (8a série) do Ensino Fundamental de escolas públicas e privadas das capitais brasileiras e do Distrito Federal.

\section{Amostragem}

Os parâmetros considerados para o cálculo do tamanho da amostra foram utilizados de modo a serem obtidas as estimativas de prevalência de algumas características de interesse, em cada um dos 27 estratos geográficos, com erro relativo máximo de 3\%, nível de 95\% de confiança. Levando-se em conta a escassez de trabalhos sobre a prevalência da maioria dos fatores de risco estudados em adolescentes, foi utilizado como parâmetro para o cálculo do tamanho amostral 
a prevalência de $50 \% 31$ de várias situações de saúde do adolescente.

O processo amostral da PeNSE foi executado em dois estágios. No primeiro, as unidades primárias de amostragem foram as escolas, de acordo com o tipo privada e pública, e a localização geográfica (26 capitais e o Distrito Federal). A amostragem das escolas foi feita por meio do método de seleção com probabilidades proporcionais ao tamanho (número total de turmas do 9o ano do Ensino Fundamental de cada escola, de acordo com o Censo Escolar 2007). No segundo estágio, as unidades secundárias de amostragem foram as turmas. Todos os alunos presentes nas salas de aula no momento da aplicação do questionário foram incluídos na amostra.

Utilizou-se uma ponderação para representar os escolares matriculados no 9o ano do Ensino Fundamental que frequentam regularmente as aulas; o fator de ponderação foi calculado considerando os estudantes ausentes no dia da pesquisa e aqueles que não responderam à variável sexo 6 .

\section{Coleta de dados}

O questionário incluiu os seguintes temas: alimentação, tabagismo, consumo de álcool e outras drogas, acidentes, violência e outros. A coleta de dados foi realizada entre os meses de março e junho de 2009, por meio de questionários padronizados autoaplicavéis, com múltipla escolha de respostas preenchidas em microcomputadores de mão (Personal Digital Assistant - PDA) 32 . Esses questionários foram elaborados de acordo com os instrumentos validados utilizados em outros estudos, como o Health Behaviour in Schoolaged Children ${ }^{3}$, o Youth Risk Behavior Surveillance Systems 25, Vigilância de Fatores de Risco para Doenças Não Transmissíveis entre Adolescentes 33, Vigescola 34. Além disso, foram realizados pré-testes com adolescentes do 9o ano em oito escolas (públicas e privadas) em Belém (Pará), Luziânia (Goiás), Mesquita (Rio de Janeiro), Recife (Pernambuco) e Rio de Janeiro para avaliar a compreensão e adequação da linguagem utilizada, manuseio do equipamento e tempo de resposta. Os pré-testes indicaram boa aceitação da pesquisa e do uso de equipamento.

\section{Variáveis estudadas}

A violência física foi considerada de acordo com a resposta à pergunta: "Nos últimos 30 dias, você esteve envolvido(a) em alguma briga em que alguém foi fisicamente agredido?", sendo analisada como variável dependente e definida como "envolvimento em situações de violência física, como agressor ou vítima, nos últimos 30 dias". Esse desfecho foi categorizado em Sim $=1$ e Não $=0$.

Os fatores potencialmente associados ao envolvimento em situações de violência física avaliados neste trabalho contemplaram as seguintes questões da PeNSE:

a) Consumo de álcool: "Nos últimos 30 dias, quantos dias você tomou pelo menos um copo ou uma dose de bebida alcoólica?";

b) Uso de drogas ilícitas: "Nos últimos 30 dias, quantas vezes você usou drogas tais como maconha, cocaína, crack, cola, loló, lança perfume, ecstasy, etc.?”. As opções de respostas para essas perguntas estavam dispostas em sete categorias relativas aos últimos 30 dias anteriores ao estudo: nenhum dia, 1 ou 2, 3-5, 6-9, 10-19, 20-29 e todos os 30 dias. Essas variáveis foram agrupadas em duas categorias $[\mathrm{Sim}=1$ (pelo menos um dia) e Não = 0 (nenhum dia)] devido à impossibilidade de relacionar temporalmente estas exposições com o desfecho;

c) Bullying: "Nos últimos 30 dias, com que frequência algum dos seus colegas de sua escola lhe esculacharam, zoaram, mangaram, intimidaram ou caçoaram tanto que você ficou magoado/incomodado/aborrecido/ ofendido/humilhado?". As opções de respostas eram referentes aos últimos 30 dias: nenhuma vez (referência), raramente (1), às vezes (2), na maior parte das vezes (3) e sempre (4). Considerou-se bullying a somatória das opções 2, 3,4;

d) Moradia dos pais na mesma residência do adolescente (nenhum dos dois = referência, apenas com a mãe $=1$, somente com o pai $=2$, ambos $=3$ ).

Para a análise dos fatores (c) e (d), foram consideradas as mesmas opções de resposta da pergunta original neste estudo. As variáveis escolaridade materna (até 8 anos de estudos: esta faixa foi tratada como referência na análise, 9-11 ou $\geq 12$ anos de estudos) e tipo de escola (pública $=1$ e privada $=0$ ) mesmo que não se apresentem como significantes, foram mantidas no modelo e utilizadas como controle de confundimento relativo ao nível socioeconômico. O questionário apresentava referência à escolaridade materna, portanto esta e o tipo de escola foram considerados para representar a situação socioeconômica. Para testar a modificação de efeito no modelo de regressão logística entre o consumo de álcool e drogas ilícitas e o envolvimento do adolescente em situações de violência física, foi criada uma nova variável "modificação de efeito" com as seguintes categorias: Não consumo de álcool e de drogas (tratada como referência), e consumo de álcool e de drogas (1). 


\section{Análise estatística}

Calculou-se a prevalência e intervalo de $95 \%$ de confiança (IC95\%) para as variáveis estudadas, estratificadas por sexo com o propósito comparativo. Para verificar as associações entre o desfecho e as variáveis explanatórias, foi realizada análise bruta por meio do teste qui-quadrado e análise múltipla usando-se a regressão logística incluindo no modelo as variáveis de ajuste escolaridade da mãe e tipo de escola, conforme literatura 4,5,19. Após a análise bruta, as variáveis com um valor de $\mathrm{p} \leq 0,20$ permaneceram no modelo. Na análise múltipla, foram considerados significativos os valores de $\mathrm{p}<0,05$ para os fatores associados com o desfecho de violência familiar. As análises dos dados foram realizadas no software Stata versão 11.0 (Stata Corp., College Station, Estados Unidos), utilizando o comando survey para amostra complexa.

\section{Aspectos éticos}

A PeNSE obteve aprovação na Comissão Nacional de Ética em Pesquisa (CONEP) do Ministério da Saúde, sob a emenda no. 005/2009 referente ao Registro no. 11.537, em 10 de junho de 2009. Houve contato prévio à pesquisa com a direção das escolas amostradas e com as secretarias estaduais e municipais de Saúde e Educação. O adolescente foi convidado a participar voluntariamente da pesquisa e poderia não responder, responder parcial ou totalmente ao questionário. A participação do escolar foi voluntária e a confidencialidade das informações foi garantida. Os dados foram sigilosamente armazenados em base de dados usada exclusivamente para o propósito do estudo.

\section{Resultados}

Foram amostradas 1.453 escolas e 2.175 turmas, nas quais havia 72.872 alunos matriculados e um total de 68.735 alunos frequentes. Na data da pesquisa estavam presentes nas escolas públicas ou privadas selecionadas 63.411 adolescentes. Entretanto, foram excluídos da amostra os escolares que não concordaram em participar do estudo ( $n=501 ; 0,79 \%$ ) e aqueles que não preencheram a variável sexo $(n=2.438 ; 3,05 \%)$, resultando em uma amostra representativa de 60.973 estudantes. Desse modo, a perda amostral foi de $16,33 \%$ relativa à amostra de alunos matriculados. Os motivos da recusa não foram mensurados (Figura 1).

Entre os adolescentes entrevistados, a mediana de idade foi de 14 anos (intervalo de 13 a 15 anos), a maioria ( $\mathrm{n}=32.550 ; 52,5 \%$ ) era do sexo feminino e estudava em escolas da rede pública $(\mathrm{n}=46.441 ; 79 \%)$. Mais de $40 \%(\mathrm{n}=20.610)$ referiram a escolaridade materna de zero a oito anos de estudos e 94,8\% ( $n=53.320$ ) moravam com pelo menos um dos pais na mesma residência. A prevalência de bullying entre os escolares pesquisados foi de $31 \%$ ( $\mathrm{n}=18.398$ ), considerando os alunos que responderam que se sentiram magoados/incomodados/aborrecidos/ofendidos/ humilhados raramente, às vezes, na maior parte das vezes e sempre nos últimos 30 dias anteriores à pesquisa. $\mathrm{O}$ consumo de bebida alcoólica foi relatado por $27,3 \%$ dos indivíduos pesquisados ( $\mathrm{n}=16.298)$ e o uso de drogas ilícitas nos últimos 30 dias foi referido por 3,3\% dos adolescentes ( $\mathrm{n}=1.954)$. A prevalência de envolvimento em situações de violência física no mês anterior à pesquisa foi observada em $12,9 \%$ dos escolares ( $\mathrm{n}=7.661)$ (Tabela 1).

A frequência de envolvimento em situações de violência foi de $17,5 \%$ para o sexo masculino e $8,9 \%$ para o feminino, sendo estatisticamente diferente. Não houve diferença entre os sexos quanto ao tipo de escola e prevalência de consumo de bebida alcoólica (valores de p > 0,05). Entretanto, foram identificadas diferenças estatisticamente significativas entre as prevalências das seguintes características em ambos os sexos: escolaridade materna, moradia dos pais na mesma residência do adolescente, sofrer bullying e uso de drogas ilícitas nos últimos 30 dias prévios à pesquisa. Com relação à escolaridade materna, $43,6 \%$ dos escolares do sexo feminino e $38,9 \%$ do masculino afirmaram que suas mães apresentavam de zero a oito anos de estudos. Adolescentes do sexo masculino apresentaram maiores prevalências de uso de drogas ilícitas e bullying quando comparados com adolescentes do sexo feminino (Tabela 1).

$\mathrm{Na}$ análise bruta, o desfecho envolvimento em situações de violência física nos 30 dias prévios ao estudo foi associado com bullying, consumo de bebida alcoólica e uso de drogas ilícitas em ambos os sexos. Todavia, foi fator de proteção morar com ambos os pais e o envolvimento em situações de violência física no sexo feminino. Após o ajuste por escolaridade materna, tipo de escola e as variáveis independentes testadas, o envolvimento em situações de violência física manteve associação significativa com bullying, consumo de bebida alcoólica e uso de drogas tanto para o sexo masculino quanto para o feminino (Tabela 2).

Morar somente com o pai ou com ambos os genitores na mesma residência foi fator de proteção para o envolvimento em situações de violência física apenas no sexo feminino (OR ajustada 
Esquema representativo da amostra dos adolescentes pesquisados. Pesquisa Nacional de Saúde do Escolar (PeNSE), Brasil, 2009.

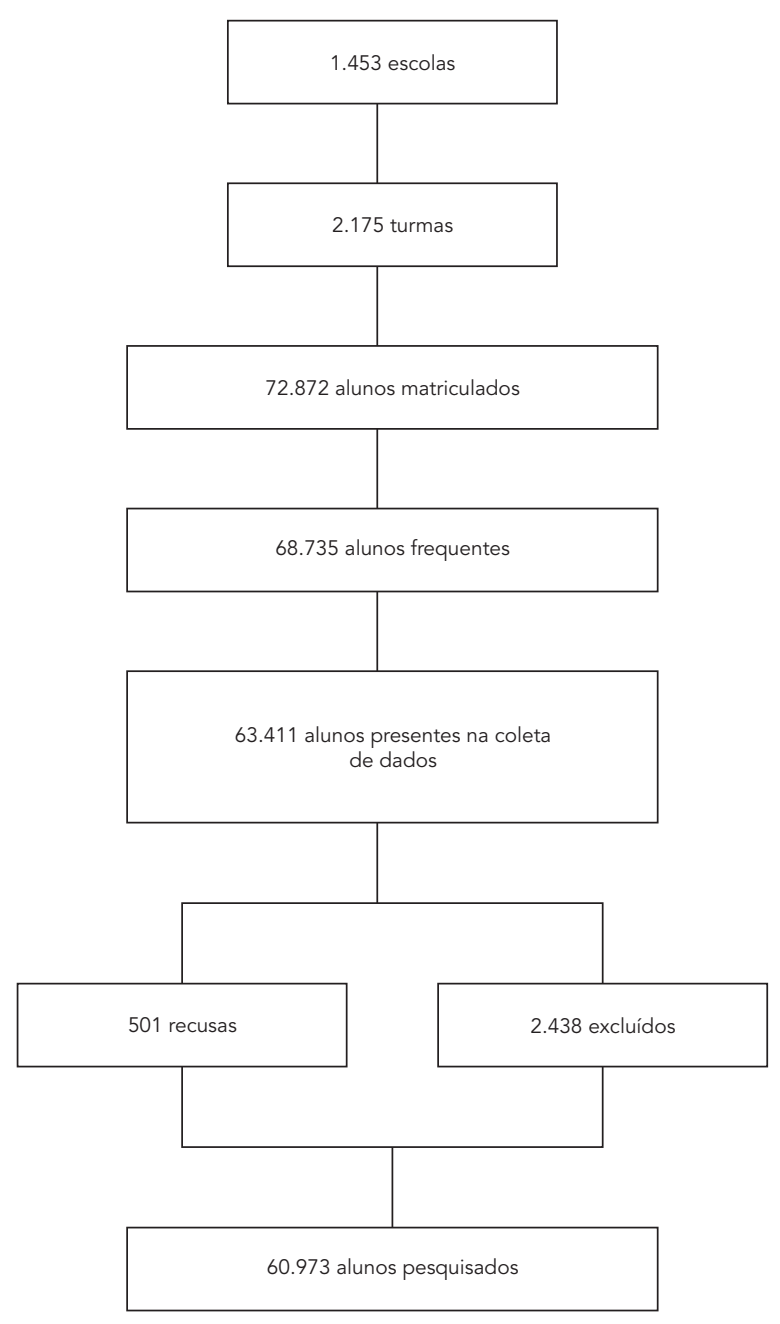

morar somente com o pai $=0,59$, IC95\%: 0,390,89 e OR ajustada morar com ambos os genitores $=0,57$, IC95\%: 0,42-0,77). Nos dois sexos, a frequência de ter sofrido bullying foi relacionada ao envolvimento em situações de violência física, exceto para o sexo masculino em que não houve diferença para a categoria raramente sofreu bullying no último mês quando comparada a nenhuma vez (OR ajustada = 1,20, IC95\%: 1,00-1,43) (Tabela 2).

Observou-se a associação entre o consumo de bebida alcoólica e o envolvimento em situações de violência física no sexo masculino (OR ajusta$\mathrm{da}=2,21$, IC95\%: 1,12-4,39), entretanto, esta re- lação não foi significante no feminino (OR ajustada $=1,30$, IC95\%: 0,62-2,71). O uso de drogas ilícitas apresentou OR ajustada de 2,75 (IC95\%: 2,30-3,30) para o sexo masculino e 3,17 (IC95\%: 2,17-4,62) para o feminino, com diferenças estatisticamente significativas em ambos os sexos.

O modelo com inclusão do termo de modificação de efeito entre consumo de álcool e drogas ilícitas é apresentado na Tabela 2. Há um sinergismo na magnitude da OR ajustada quando comparada a referência (não consumo de álcool e de drogas) com a categoria de consumo de álcool e de drogas juntamente. A OR ajustada para o efeito do consumo de álcool e drogas conjunta- 
Características dos adolescentes do 9ㅇ ano do Ensino Fundamental estratificadas por sexo. Pesquisa Nacional de Saúde do Escolar (PeNSE), Brasil, 2009.

\begin{tabular}{|c|c|c|c|c|}
\hline Variáveis & Total (\%) & Masculino (\%) & Feminino (\%) & Valor de $\mathrm{p}$ \\
\hline Total & & 47,5 & 52,5 & \\
\hline Tipo de escola [n $=60.973]$ & & & & 0,246 \\
\hline Pública & 79,2 & 78,7 & 79,6 & \\
\hline Privada & 20,8 & 21,3 & 20,4 & \\
\hline Escolaridade materna (anos de estudo) [n = 49.782] & & & & $<0,001$ \\
\hline $0-8$ & 41,4 & 38,9 & 43,6 & \\
\hline $9-11$ & 33,4 & 33,6 & 33,2 & \\
\hline 12 e mais & 25,2 & 27,5 & 23,2 & \\
\hline Moradia dos pais na residência $[n=60.464]$ & & & & $<0,001$ \\
\hline Nenhum dos pais & 5,2 & 4,6 & 5,8 & \\
\hline Apenas com a mãe & 31,9 & 29,6 & 33,9 & \\
\hline Somente com o pai & 4,6 & 5,4 & 3,9 & \\
\hline Ambos & 58,3 & 60,5 & 56,4 & \\
\hline Bullying $[\mathrm{n}=59.348]$ & & & & 0.009 \\
\hline Nenhuma vez & 69,2 & 67,4 & 70,8 & \\
\hline Raramente & 16,7 & 17,3 & 16,1 & \\
\hline Às vezes & 8,8 & 9,3 & 8,3 & \\
\hline Na maior parte das vezes & 2,7 & 2,8 & 2,6 & \\
\hline Sempre & 2,8 & 3,3 & 2,3 & \\
\hline Consumo de bebida alcoólica $[n=59.699]$ & & & & 0.097 \\
\hline Não & 72,7 & 73,5 & 71,9 & \\
\hline Sim & 27,3 & 26,5 & 28,1 & \\
\hline Uso de drogas ilícitas [ $\mathrm{n}=59.208$ ] & & & & $<0,001$ \\
\hline Não & 96,7 & 95,6 & 97,7 & \\
\hline Sim & 3,3 & 4,4 & 2,3 & \\
\hline Violência física [ $n=59.388]$ & & & & $<0,001$ \\
\hline Não & 87,1 & 82,5 & 91,1 & \\
\hline Sim & 12,9 & 17,5 & 8,9 & \\
\hline
\end{tabular}

mente foi de 7,01 (IC95\%: 5,47-8,90) para o sexo masculino e de 8,50 (IC95\%: 5,82-12,42) para o feminino.

\section{Discussão}

A prevalência de violência física entre os adolescentes pesquisados foi de $12,9 \%$ para os últimos 30 dias. Outros estudos conduzidos com inquéritos, realizados entre escolares de outros países também estimaram altas taxas de prevalência de participação em brigas com agressão física em um período de 12 meses: 13,5\% nos Estados Unidos, 31,3\% na Argentina, 31,1\% na Venezuela, $42 \%$ na Turquia, $40,7 \%$ no Chile $4,35,36,37,38$.

Neste trabalho, a prevalência de envolvimento em situações de violência física, seja como agressor ou como vítima, foi maior entre os ado- lescentes do sexo masculino, assim como relatado em outros estudos 1,3,17,27,35,38,39,40,41. O sexo masculino é apontado como preditor de respostas violentas em situações de confronto ou vitimização 27. Uma possível explicação para esse fato é que o comportamento agressivo é tolerado e muitas vezes estimulado em sociedades com dominação de padrões culturais machistas 27,35,39. Por outro lado, as meninas tendem a desenvolver mais estresse pós-traumático e depressão após exposição a situações de violência 33 .

O tipo de escola e a escolaridade materna não foram associados com os episódios de violência física entre os escolares nesta pesquisa. Estudo sobre os fatores de proteção envolvendo a família de adolescentes e comportamentos de risco e prevalência de participação em brigas com agressão física, também não evidenciaram diferença entre escola pública e privada 28,37 . 
Fatores associados com o envolvimento em situações de violência física e variáveis selecionadas, estratificadas por sexo. Brasil. Pesquisa Nacional de Saúde do Escolar (PeNSE), Brasil, 2009.

\begin{tabular}{|c|c|c|c|c|}
\hline \multirow[t]{2}{*}{ Variável } & \multicolumn{2}{|c|}{ Sexo masculino } & \multicolumn{2}{|c|}{ Sexo feminino } \\
\hline & OR bruta (IC95\%) & $\begin{array}{l}\text { OR ajustada } \\
\text { (IC95\%) }\end{array}$ & OR bruta (IC95\%) & $\begin{array}{l}\text { OR ajustada * } \\
\text { (IC95\%) }\end{array}$ \\
\hline \multicolumn{5}{|l|}{ Tipo de escola } \\
\hline Pública & Referência & Referência & Referência & Referência \\
\hline Particular & $1,05(0,92-1,20)$ & $0,92(0,79-1,07)$ & $0,81(0,66-1,00)$ & $0,92(0,69-1,23)$ \\
\hline \multicolumn{5}{|l|}{ Escolaridade materna (anos de estudo) } \\
\hline $0-8$ & Referência & Referência & Referência & Referência \\
\hline $9-11$ & $1,04(0,90-1,20)$ & $1,04(0,91-1,19)$ & $0,99(0,85-1,14)$ & $1,07(0,93-1,24)$ \\
\hline 12 e mais & $1,04(0,92-1,19)$ & $1,06(0,94-1,19)$ & $0,88(0,76-1,02)$ & $0,95(0,75-1,22)$ \\
\hline \multicolumn{5}{|c|}{ Moradia de pelo menos um dos pais na residência } \\
\hline Nenhum dos pais & Referência & Referência & Referência & Referência \\
\hline Apenas com a mãe & $1,17(0,89-1,53)$ & $1,14(0,85-1,53)$ & $0,82(0,58-1,15)$ & $0,74(0,50-1,11)$ \\
\hline Somente com o pai & $1,30(0,93-1,82)$ & $1,22(0,79-1,90)$ & $0,66(0,39-1,11)$ & $0,59(0,39-0,89)$ \\
\hline Ambos & $0,94(0,81-1,10)$ & $0,97(0,79-1,21)$ & $0,57(0,43-0,76)$ & $0,57(0,42-0,77)$ \\
\hline \multicolumn{5}{|l|}{ Bullying } \\
\hline Nenhuma vez & Referência & Referência & Referência & Referência \\
\hline Raramente & $1,31(1,15-1,49)$ & $1,20(1,00-1,43)$ & $1,46(1,32-1,62)$ & $1,37(1,17-1,61)$ \\
\hline Às vezes & $1,37(1,20-1,56)$ & $1,38(1,45-1,66)$ & $1,76(1,41-2,19)$ & $1,97(1,59-2,45)$ \\
\hline Na maior parte das vezes & $1,80(1,12-2,88)$ & $1,79(1,11-2,88)$ & $1,79(1,40-2,30)$ & $1,98(1,37-2,86)$ \\
\hline Sempre & $2,29(1,83-2,85)$ & $2,11(1,56-2,85)$ & $3,87(3,02-4,97)$ & $3,84(2,76-5,36)$ \\
\hline \multicolumn{5}{|l|}{ Consumo de bebida alcoólica } \\
\hline Não & Referência & Referência & Referência & Referência \\
\hline Sim & $2,98(2,64-3,36)$ & $2,21(1,12-4,39)$ & $2,88(2,56-3,24)$ & $1,30(0,62-2,71)$ \\
\hline \multicolumn{5}{|l|}{ Uso de drogas ilícitas } \\
\hline Não & Referência & Referência & Referência & Referência \\
\hline Sim & $4,83(3,77-6,21)$ & $2,75(2,30-3,30)$ & $5,89(4,04-8,59)$ & $3,17(2,17-4,62)$ \\
\hline \multicolumn{5}{|l|}{ Modificação de efeito } \\
\hline Não consumo álcool e drogas ilícitas & Referência & Referência & Referência & Referência \\
\hline Consumo de álcool e drogas ilícitas & $5,22(4,16-6,56)$ & $7,01(5,47-8,90)$ & $5,86(3,90-8,82)$ & $8,50(5,82-12,42)$ \\
\hline
\end{tabular}

IC95\%: intervalo de 95\% de confiança; OR: odds ratio.

* Modelo ajustado para todas as variáveis.

No nosso trabalho, não houve associação entre o envolvimento do adolescente com violência física e residir com pelo menos um dos pais no sexo masculino. Todavia, essas variáveis mostraram-se inversamente associadas para o sexo feminino, isto é, morar com pelo menos um dos genitores foi indicativo de fator de proteção no sexo feminino para situações de violência física. A análise da PeNSE em relação aos fatores familiares mostrou associação entre não morar com os pais e maior risco de fatores como tabaco, álcool e drogas em ambos os sexos 26. Um estudo com 960 adolescentes em Pelotas mostrou que não houve associação entre o envolvimento em brigas e coabitar com pelo menos um dos pais. Os autores reforçaram a importância de considerar o impacto da coabitação parento-filial e suas consequências quando os filhos vivem na mesma residência que qualquer um dos pais 42 .

Entretanto, a diferença encontrada nessa associação para meninos e meninas pode ser decorrente da exposição à violência diferenciada entre os sexos 39; além disto, o ensino de um comportamento violento socialmente aceito na família é diferente para meninos e meninas 40 . A família exerce um papel fundamental na promoção de respostas não violentas e em desencorajar comportamentos agressivos entre os adolescentes na resolução de conflitos entre os pares 43 . 
Neste estudo, problemas com o consumo de álcool e drogas foi significantemente associado com a violência física entre os adolescentes do sexo masculino, corroborando com os dados da literatura 2,4,38,44. O uso de álcool e de drogas ilícitas foi considerado preditor de comportamentos violentos entre adolescentes e associado a outras consequências negativas à saúde 10,22,43,44,45.

Não houve diferença entre os sexos na prevalência de consumo de álcool, entretanto, esta diferença foi identificada para o uso de drogas ilícitas - meninos apresentaram maior prevalência do que as meninas. Todavia, diversos estudos mostraram que os meninos consomem mais bebidas alcoólicas do que as meninas 42,44,46,47, e adolescentes do sexo feminino apresentam menor consumo de drogas ilícitas quando comparadas com o sexo masculino ${ }^{23}$. A prevalência de uso de drogas ilícitas foi considerada pequena nos estudos 22,23, e Vieira et al. 22 argumentam que os escolares podem omitir experiências passadas sobre este uso.

Neste trabalho, apesar da diferença não ser significativa, a magnitude dessas relações entre consumo de álcool e drogas ilícitas com envolvimento em situações de violência física é maior entre os meninos considerando consumo de álcool, e entre as meninas quanto ao uso de drogas. Nossas hipóteses são de que o consumo dessas substâncias funciona como estimulante para os adolescentes assumirem um comportamento mais agressivo e com maior envolvimento em situações de violência. Young et al. ${ }^{21}$ apontaram que o comportamento antissocial (inclui dentre outros fatores, brigar, assumir riscos e "quebrar" regras) é um fator que predispõe o consumo/ abuso de álcool entre adolescentes em contraposição à hipótese de que o uso de álcool ocasiona comportamentos antissociais - chamados efeito de desinibição.

Entre os fatores de risco associados ao consumo de álcool e drogas ilícitas por adolescentes são descritos tabagismo, uso de drogas, consumo de bebidas alcoólicas pelos pais, baixa escolaridade, classe social baixa, frágil vínculo escolar, ocorrência de reprovações escolares, influência de amigos e colegas, e comportamento antissocial 21,43,46,48. Um importante resultado do presente estudo foi que o uso conjunto de álcool e drogas ilícitas potencializou a chance de envolvimento em situações de violência para adolescentes de ambos os sexos. Todavia, salienta-se que comportamentos de risco como consumo de álcool e uso de drogas podem ser motivadores para o envolvimento em situações de violência física 49 ou resultantes da vitimização do adolescente por meio da violência interpessoal 50 .
Para os adolescentes pesquisados, o envolvimento em situações de violência física também manteve associação com bullying, não sendo observadas diferenças entre os sexos. Ser vítima de bullying eleva a probabilidade de envolvimento em situações de violência física entre adolescentes, independentemente do sexo, tabagismo, consumo de álcool/drogas e supervisão dos pais 37,38. Os fatores que influenciam o comportamento violento nessas circunstâncias incluem pouco controle emocional, baixa autoestima, histórico de envolvimento em situações de violência, problemas no desempenho escolar, estímulo dos pais à reação violenta dos adolescentes quando vítimas de bullying, pressão dos colegas e amigos para assumir comportamentos violentos e o próprio bullying 18,20,27,37,43.

Salienta-se que, nas situações de bullying, é necessária a atuação do professor como mediador, pois quando o estudante sente-se seguro e apoiado sua atitude tende a não ser violenta quando vitimizado 27,43. Os fatores apontados neste trabalho como independentemente associados à violência física entre adolescentes foram: consumo de álcool, uso de drogas ilícitas e sofrer bullying. Estudos semelhantes como o Inquérito Global Chileno de Saúde Escolar - Santiago, Inquérito Global Venezuelano de Saúde Escolar - Barinas, e Health Behaviour in School-Aged Children (coordenado pela OMS e que incluiu 35 países da Europa, Oriente Médio e América do Norte) indicaram resultados similares 4,36,51.

Entre as limitações deste estudo, pode-se destacar a pergunta do questionário sobre violência física ("Nos últimos 30 dias, você esteve envolvido (a) em alguma briga em que alguém foi fisicamente agredido?") que não possibilita identificar o adolescente como vítima ou agressor. O conceito subjetivo de violência pode ter influenciado o entendimento das questões e, consequentemente, suas respostas. O tipo de escola e a escolaridade materna podem não ter sido suficientes para avaliar a diferença entre nível socioeconômico e envolvimento em situações de violência física. Dessa forma, sugere-se que outras variáveis, como posse de bens, sejam utilizadas para avaliar a relação entre nível socioeconômico e violência física entre adolescentes, e também questões que identifiquem se o adolescente foi vítima e/ ou agressor.

O cálculo amostral da PeNSE foi executado segundo a prevalência de $50 \%$ de várias situações de saúde do adolescente, isto é uma limitação, pois como os dados deste artigo foram extraídos daquela pesquisa mais ampla, não foi possível realizar o cálculo amostral a partir de nosso desfecho clínico de violência física que seria, segundo estudos brasileiros, entre $12,9 \%$ e $29,2 \%$. Outra 
limitação refere-se à impossibilidade de realizar inferências causais/temporais entre exposições avaliadas, pois trata-se de estudo transversal, não sendo possível avaliar se o uso de álcool ou consumo de drogas ilícitas é causa ou consequência do envolvimento do adolescente em situações de violência física.

Uma limitação da pesquisa foi a inclusão apenas de adolescentes que estavam frequentando a escola e presentes em sala de aula no momento da aplicação do questionário. Decisão que pode ter ocasionado algum viés nos resultados, pois o absenteísmo ou evasão escolar está relacionado a fatores estudados como consumo de álcool, uso de drogas ilícitas, situações de violência, entre outros 25,26,32. Entretanto, essa limitação não inviabiliza os resultados, pois, o trabalho foi realizado com uma amostra expressiva. Ressaltase que as cidades do interior podem apresentar realidade diferente da observada.

Outros estudos são necessários, especialmente análises qualitativas, para entendimento aprofundado da violência entre os adolescentes. Recomenda-se também que os programas de prevenção da violência e promoção da cultura de paz entre os adolescentes considerem as diferentes experiências de violência entre os sexos, e atuem de maneira distinta para que sejam mais eficazes, criando ambientes favoráveis 43,46,52. O papel da escola na saúde dos adolescentes é endossado pelo Programa Saúde na Escola (PSE), instituído em 2007, e que tem entre os seus principais objetivos a promoção da saúde e da cultura de paz, e a contribuição para o enfrentamento das vulnerabilidades em saúde que comprometem o desenvolvimento escolar, articulando os setores da saúde e educação 53 .

A violência física entre os adolescentes é um marcador de outros estilos de vida não saudáveis, possivelmente não numa relação do tipo causa/ efeito, mas devido ao fato de que um adolescente exposto a um estilo de vida não saudável pode estar submetido a outros fatores de risco ou agravos contra sua saúde 5 . Os resultados desta pesquisa mostram que o envolvimento em situações de violência física é mais prevalente no sexo masculino e que o consumo de álcool, o uso de drogas ilícitas e ser vítima de bullying estão associados à violência física entre os adolescentes. Esses dados indicam a necessidade de ações de promoção da saúde e cultura da paz na adolescência, de modo a contribuir para romper a ideia de que a violência entre adolescentes é algo banal e esperado.

\section{Resumo}

O objetivo deste estudo foi identificar a associação entre o consumo de álcool e outras drogas e o bullying com o envolvimento em situações de violência física entre adolescentes de 13 a 15 anos, em escolas públicas e privadas das capitais brasileiras e do Distrito Federal. Foram analisados os dados da Pesquisa Nacional de Saúde do Escolar (PeNSE) de 2009. Para análise dos dados foi utilizada a regressão logística. A prevalência de envolvimento em situações de violência física foi 12,9\% maior no sexo masculino. Em ambos os sexos, foram observadas associações entre violência física e ser vítima de bullying com o uso de drogas ilícitas e efeito potencializado do consumo de álcool e drogas.
Para o sexo masculino, o uso de álcool mostrou associação significante com violência física. Morar o pai ou ambos os genitores na residencia apresentou associação inversa para violência física no sexo feminino. O conhecimento de fatores associados à violência física entre adolescentes é importante para auxiliar estratégias de promoção da saúde e da cultura de paz, rompendo com a ideia de que a violência entre adolescentes é algo banal e esperado.

Violência; Adolescente; Consumo de Bebidas Alcoólicas; Drogas Ilícitas; Bullying 


\section{Colaboradores}

S. S. C. A. Andrade contribuiu na escrita, revisão da literatura, análise estatística e interpretação dos dados, e revisão crítica do manuscrito. R. T. C. Yokota contribuiu na análise estatística e revisão crítica do manuscrito. N. N. B. SÁ colaborou na revisão da literatura e revisão final do manuscrito. M. M. A. Silva participou na revisão crítica do manuscrito e interpretação dos dados. W. N. Araújo auxiliou na interpretação dos dados e revisão crítica do manuscrito. M. D. M. Mascarenhas contribuiu na revisão de literatura e revisão crítica do manuscrito. D. C. Malta auxiliou na interpretação dos dados, revisão da literatura e revisão crítica final do manuscrito.

\section{Agradecimentos}

Agradecemos à professora Suely Tuboi pelo auxílio na revisão da escrita deste texto.

\section{Referências}

1. World Health Organization. World report on violence and health: summary. Geneva: World Health Organization; 2002.

2. Silva RA, Jansen K, Godoy RV, Souza LDM, Horta BL, Pinheiro RT. Prevalência e fatores associados a porte de arma e envolvimento em agressão física entre adolescentes de 15 a 18 anos: estudo de base populacional. Cad Saúde Pública 2009; 25: 2737-45.

3. World Health Organization. Inequalities in young people's health. Health behavior in school-aged children international report from the 2005/2006 survey. Copenhagen: World Health Organization; 2008.

4. Smith-Khuri E, Iachan R, Scheidt PC, Overpeck MD, Gabhainn SN, Pickett W, et al. A cross-national study of violence-related behaviors in adolescents. Arch Pediatr Adolesc Med 2004; 158:539-44.

5. Rudatsikira E, Muula AS, Siziya S. Prevalence and correlates of physical fighting among school-going adolescents in Santiago, Chile. Rev Bras Psiquiatr 2008; 30:197-202.
6. Instituto Brasileiro de Geografia e Estatística. Pesquisa Nacional de Saúde do Escolar - PeNSE: 2009. Rio de Janeiro: Instituto Brasileiro de Geografia e Estatística; 2009.

7. Jacobson LSV, Andrade CLT, Carmo CN, Mourão DS, Hacon SS. Trend in mortality due to external causes in the State of Espírito Santo, Brazil, from 1994 to 2005. Rev Bras Epidemiol 2009; 12:82-91.

8. Villela LCM, Moraes SA, Suzuki CS, Freitas ICM. Tendência da mortalidade por homicídios em Belo Horizonte e região metropolitana: 1980-2005. Rev Saúde Pública 2010; 44:486-95.

9. Araújo EM, Costa MCN, Oliveira NF, Santana FS, Barreto ML, Hogan V, et al. Spatial distribution of mortality by homicide and social inequalities according to race/skin color in an intra-urban Brazilian space. Rev Bras Epidemiol 2010; 13:549-60.

10. Departamento de Análise de Situação de Saúde, Secretaria de Vigilância em Saúde, Ministério da Saúde. Viva: vigilância de violências e acidentes, 2008 e 2009. Brasília: Ministério da Saúde; 2010. (Série G. Estatística e Informação em Saúde). 
11. Meneghel SN, Giugliani EJ, Falceto O. Relações entre violência doméstica e agressividade na adolescência. Cad Saúde Pública 1998; 14:327-35.

12. Assis SG, Avanci JQ, Santos NC, Malaquias JV, Oliveira RVC. Violência e representação social na adolescência no Brasil. Rev Panam Salud Pública 2004; 16:43-51.

13. Bordin IA, Duarte CS, Peres CA, Nascimento R, Curto BM, Paula CS. Severe physical punishment: tisk of mental health problems for poor urban children in Brazil. Bull World Health Organ 2009; 87:336-44.

14. Brito AMM, Zanetta DMT, Mendonça RCV, Barison SZP, Andrade VAG. Violência doméstica contra crianças e adolescentes: estudo de um programa de intervenção. Ciênc Saúde Coletiva 2005; 10:143-9.

15. Zavaschi ML, Benetti S, Polanczyk GV, Solés N, Sanchotene ML. Encuesta en escuelas públicas de Brasil sobre la exposición de los adolescentes a la violencia en la comunidad. Rev Panam Salud Pública 2002; 12:327-32.

16. Carlini-Cotrim B, Gazal-Carvalho C, Gouveia N. Comportamentos de saúde entre jovens estudantes das redes pública e privada da área metropolitana do Estado de São Paulo. Rev Saúde Pública 2000; 34:636-45.

17. Cruzeiro ALS, Silva RA, Horta BL, Souza LDM, Faria AD, Pinheiro RT, et al. Prevalência e fatores associados ao transtorno da conduta entre adolescentes: um estudo de base populacional. Cad Saúde Pública 2008; 24:2013-20.

18. Resnick MD, Ireland M, Borowsky I. Youth violence perpetration: what protects? What predicts? Findings from the National Longitudinal Study of adolescent health. J Adolesc Health 2004; 35:424. e1-10.

19. Benetti SPC, Pizetta A, Schwartz CB, Hass RA, Melo VL. Problemas de saúde mental na adolescência: características familiares, eventos traumáticos e violência. Psico USF 2010; 15:321-32.

20. Youngblade LM, Theokas C, Schulenberg J, Curry L, Huang IC, Novak M. Risk and promotive factors in families, schools, and communities: a contextual model of positive youth development in adolescence. Pediatrics 2007; 119 Suppl 1:S47-53.

21. Young R, Sweeting H, West P. A longitudinal study of alcohol use and antisocial behaviour in young people. Alcohol Alcohol 2008; 43:204-14.

22. Vieira PC, Aerts DRGC, Freddo SL, Bittencourt A, Monteiro L. Uso de álcool, tabaco e outras drogas por adolescentes escolares em município do Sul do Brasil. Cad Saúde Pública 2008; 24:2487-98.

23. Horta RL, Horta BL, Pinheiro RT, Morales B, Strey MN. Tabaco, álcool e outras drogas entre adolescentes em Pelotas, Rio Grande do Sul, Brasil: uma perspectiva de gênero. Cad Saúde Pública 2007; 23:775-83.

24. Moore GF, Rothwell H, Segrott J. An exploratory study of the relationship between parental attitudes and behaviour and young people's consumption of alcohol. Subst Abuse Treat Prev Policy 2010; 5:6.
25. Galduróz JCF, Noto AR, Fonseca AM, Carlini EA. $\mathrm{V}$ levantamento nacional sobre o consumo de drogas psicotrópicas entre estudantes do Ensino Fundamental e Médio da rede pública de ensino nas 27 capitais brasileiras - 2004. http://www.uni fesp.br/dpsicobio/cebrid/levantamento_brasil2/ index.htm (acessado em Out/2011).

26. Malta DC, Mascarenhas MDM, Porto DL, Duarte EA, Sardinha LM, Barreto SM, et al. Prevalência do consumo de álcool e drogas entre adolescentes: análise dos dados da Pesquisa Nacional de Saúde Escolar. Rev Bras Epidemiol 2011; 14 Suppl 1: S136-46.

27. Aceves MJ, Hinshaw SP, Mendoza-Denton R, PageGould E. Seek help from teachers or fight back? Student perceptions of teachers' actions during conflicts and responses to peer victimization. J Youth Adolesc 2010; 39:658-69.

28. Malta DC, Porto DL, Melo FCM, Monteiro RA, Sardinha LMV, Lessa BH. Família e proteção ao uso de tabaco, álcool e drogas em adolescentes, Pesquisa Nacional de Saúde dos Escolares. Rev Bras Epidemiol 2011; 14 Suppl 1:166-77.

29. Paula CS, Vedovato MS, Bordin IA, Barros MG, D'Antino ME, Mercadante MT. Mental health and violence among sixth grade students from a city in the state of São Paulo. Rev Saúde Pública 2008; 42:524-8.

30. World Health Organization. Global school-based student health survey (GSHS) purpose and methodology. http://www.who.int/chp/gshs/method ology/en/index.html (acessado em Out/2011).

31. Luiz RR, Magnanini MMF. O tamanho da amostra em investigações epidemiológicas. In: Medronho RA, Bloch KV, Luiz RR, Werneck GL, organizadores. Epidemiologia. São Paulo: Editora Atheneu; 2009. p. $415-27$.

32. Malta DC, Souza ER, Silva MMA, Silva CS, Andreazzi MAR, Crespo C, et al. Vivência de violência entre escolares brasileiros: resultados da Pesquisa Nacional de Saúde do Escolar (PeNSE). Ciênc Saúde Coletiva 2010; 15:3053-63.

33. Castro IRR, Cardoso LO, Engstrom EM, Levy RB, Monteiro CA. Vigilância de fatores de risco para doenças não transmissíveis entre adolescentes: a experiência da cidade do Rio de Janeiro, Brasil. Cad Saúde Pública 2008; 24: 2279-88.

34. Instituto Nacional de Câncer. Vigescola. Vigilância de tabagismo em escolares: dados e fatos de 12 capitais brasileiras. v. 1. Brasília: Instituto Nacional de Câncer; 2004.

35. Rudatsikira E, Muula AS, Siziya S. Variables associated with physical fighting among US high-school students. Clin Pract Epidemiol Ment Health 2008; 4:16.

36. Linetzky, B. Encuesta mundial de la salud escolar. Resultados de 2007. Argentina. http://www.who. int/chp/gshs/2007_GSHS_Report_ARG_ES.pdf (acessado em Abr/2012).

37. Muula AS, Herring P, Siziya S, Rudatsikira E. Bullying victimization and physical fighting among Venezuelan adolescents in Barinas: results from the Global School-Based Health Survey 2003. Ital J Pediatr 2009; 35:38. 
38. Alikasifoglu M, Erginoz E, Ercan O, Uysal O, Kaymak D, Iiter O. Violent behavior among Turkish high school students and correlates of physical fighting. Eur J Public Health 2004; 14:173-7.

39. Hanson RF, Borntrager C, Self-Brown S, Kilpatrick DG, Saunders BE, Resnick HS, et al. Relations among gender, violence exposure, and mental health: the National Survey of adolescents. Am J Orthopsychiatry 2008; 78:313-21.

40. Garbarino J. Why are adolescents violent? Ciênc Saúde Coletiva 2009; 14:533-8.

41. Centers for Disease Control and Prevention. National Youth Risk Behavior Survey (YRBS). Trends in behaviors that contribute to violence. National YRBS: 1991-2007. http://www.cdc.gov/yrbss (acessado em 15/Set/2009).

42. Horta RL, Horta BL, Pinheiro RT, Krindges M. Comportamentos violentos de adolescentes e coabitação parento-filial. Rev Saúde Pública 2010; 44:979-85.

43. Farrell AD, Mays S, Bettencourt A, Erwin EH, VulinReynolds M, Allison KW. Environmental influences on fighting versus nonviolent behavior in peer situations: a qualitative study with urban AfricanAmerican adolescents. Am J Community Psychol 2010; 46:19-35.

44. Gomes BMR, Alves JGB, Nascimento LC. Consumo de álcool entre estudantes de escolas públicas da Região Metropolitana do Recife, Pernambuco, Brasil. Cad Saúde Pública 2010; 26:706-12.

45. Swahn MH, Donovan JE. Correlates and predictors of violent behavior among adolescent drinkers. J Adolesc Health 2004; 34:480-92.

46. Parsai M, Voisine S, Marsiglia FF, Kulis S, Nieri T. The protective and risk effects of parents and peers on substance use, attitudes and behaviors of Mexican and Mexican American female and male adolescents. Youth Soc 2009; 40:353-76.
47. Carvalho PD, Barros MVG, Lima RA, Santos CM, Melo EN. Condutas de risco à saúde e indicadores de estresse psicossocial em adolescentes estudantes do Ensino Médio. Cad Saúde Pública 2011; 27:2095-105.

48. Noal RB, Menezes AMB, Araujo CL, Hallal PC. Experimental use of alcohol in early adolescence: the 11-year follow-up of the 1993 Pelotas (Brazil) birth cohort study. Cad Saúde Pública 2010; 26:1937-44.

49. Bye EK, Rossow I. The impact of drinking pattern on alcohol-related violence among adolescents: an international comparative analysis. Drug Alcohol Rev 2010; 29:131-7.

50. Danielson CK, Arellano MA, Ehrenreich JT, Suárez LM, Bennett SM, Cheron DM, et al. Identification of high-risk behaviors among victimized adolescents and implications for empirically supported psychosocial treatment. J Psychiatr Pract 2006; 12:364-83.

51. Pickett W, Craig W, Harel Y, Cunningham J, Simpson K, Molcho M, et al. Cross-national study of fighting and weapon carrying as determinants of adolescent injury. Pediatrics 2005; 116:e855-63.

52. Aerts D, Alves GG, La Salvia MW, Abegg C. Promoção de saúde: a convergência entre as propostas da vigilância da saúde e da escola cidadã. Cad Saúde Pública 2004; 20:1020-8.

53. Brasil. Decreto no. 6.286, de 5 de dezembro de 2007. Institui o Programa Saúde na Escola - PSE, e dá outras providências. Diário Oficial da União 2007; 6 dez.

Recebido em 28/Dez/2011

Versão final reapresentada em 11/Jun/2012 Aprovado em 25/Jun/2012 